Europhysics Letters

PREPRINT

\title{
Layer dependent band dispersion and correlations using tunable Soft X-ray ARPES
}

\author{
N. Kamakura ${ }^{1}$, Y. Takata $^{1}$, T. Tokushima $^{1}$, Y. Harada $^{1}$, A. Chainani $^{1,2}$, K. $^{2}$ \\ KOBAYASHI $^{3}$ and S. SHIN ${ }^{1,4}$ \\ 1 RIKEN, Harima Institute, 1-1-1 Kouto, Mikazuki, Sayo, Hyogo 679-5148, Japan \\ 2 Institute for Plasma Research, Bhat, Gandhinagar 382 428, Gujarat, India \\ 3 SPring-8/JASRI Mikazuki, Hyogo 679-5198, Japan \\ 4 Institute for Solid State Physics, University of Tokyo, Kashiwanoha, Kashiwa, Chiba \\ 27r-8581, Japan
}

PACS. 71.20.Be - Transition metals and alloys.

PACS. 79.60.-i - Photoemission and photoelectron spectra.

PACS. 75.50.Cc - Other ferromagnetic metals and alloys.

\begin{abstract}
Soft X-ray Angle-Resolved Photoemission Spectroscopy is applied to study inplane band dispersions of Nickel as a function of probing depth. Photon energies between $h \nu$ $=190$ and $780 \mathrm{eV}$ were used to effectively probe up to $\sim 3-7$ layers $(\sim 5-12 \AA)$. The results show layer dependent band dispersion of the $\Delta_{2 \downarrow}$ minority-spin band which crosses the Fermi level in 3 or more layers, in contrast to known top 1-2 layers dispersion obtained using ultraviolet rays. The layer dependence corresponds to an increased value of exchange splitting and suggests reduced correlation effects in the bulk compared to the surface.
\end{abstract}

Introduction. - Angle-Resolved Photoemission Spectroscopy (ARPES) is a valuable tool to study the experimental band-structure(BS), $w(\mathbf{k})$, where $w$ is energy and $\mathbf{k}$ is momentum of electrons in a solid. Recent Ultra-violet-ARPES (ARUPS) studies of correlated materials have provided important results like spin and charge collective modes in a quasi 1-D (dimensional) metal [1], dimensional crossover [2], exotic characteristics of the high- $T_{c}$ cuprates [3], etc. While ARUPS is extremely well suited to study electronic BS of low-dimensional solids, it probes the top 1-2 layers of the surface [4]. In order to determine bulk BS of 3-D correlated systems which can depend on the probed layer [5-8], it is meaningful to use higher energies. As the excitation energy is increased, the probing depth or mean free path(MFP) increases [9], but to date, no layer dependent variation of in-plane band dispersion (BD) beyond the top 2 layers has been reported. In this work, using tunable soft X-ray ARPES, we study layer dependence of in-plane BDs of Nickel metal.

Nickel is a prototype of a correlated ferromagnetic metal and has been extensively studied using PES and inverse-PES spectroscopies [10-19]. Beginning with the work of Slater [20] and Stoner [21], the BS of Nickel has remained a suitable testing ground for a variety of experiments and theory. Recent theories have addressed the major inconsistencies with experiment : $3 d$ bandwidth extent, temperature(T)-dependence of magnetization, and a satellite structure

(C) EDP Sciences 
which appears at about $6 \mathrm{eV}$ below the Fermi level $\left(E_{F}\right)$. The $3 d$ bandwidth and exchange splitting(ES) of Nickel observed by ARUPS are reduced by $25 \%$ and $50 \%$, respectively [1015], compared to the values obtained from spin-polarized local density approximation (sLDA) calculations [22]. The $6 \mathrm{eV}$ satellite obtained in PES experiments [16-18] is the twohole bound state due to correlation effects. It is missing in BS calculations in the oneelectron picture [22] but obtained in "generalized Hubbard models" [14,19, 23] or the "LDA (GWA) + DMFT" (Dynamical Mean-Field Theory) $[24,25]$ which explicitly include Coulomb interactions. A recent study discusses the inability of s-LDA to reproduce the position of the minority spin state $X_{2 \downarrow}$ below $E_{F}$ obtained by ARUPS [14]. The energy position of $X_{2 \downarrow}$ states determines the electron count associated with the hole pocket of minority spin character at $X$-point, which in turn influences the magnetic moment, a macroscopic property. Although recent theoretical efforts reproduce the experimental BS of Nickel by including correlation effects $[14,25]$, the results were compared with experimental BS obtained using ARUPS. The present study reports new experimental results of the bulk BS of Nickel obtained using soft X-ray ARPES.

Experiment. - Experiments were performed at beam line 27SU of SPring-8 [26] using linearly polarized light. Total energy resolution was $50-160 \mathrm{meV}$. The beam line has a figure8 undulator [27], enabling an easy switch of the polarization vector from horizontal(H)- to vertical(V)- polarization. $\mathrm{Ni}(100)$ surface was prepared by $\mathrm{Ar}^{+}$sputtering and annealing. The surface was checked by core level photoemission spectra obtained using $780 \mathrm{eV}$ photons and the contamination due to Oxygen and Carbon was measured to be $<1 \%$. The surface crystallinity was confirmed to be a sharp $(1 \times 1)$ LEED pattern.

ARPES measures $w$ and $\mathbf{k}$ of electrons in a solid according to the following equations [4].

$$
\hbar \mathbf{k}_{\|}=\sqrt{2 m(h \nu-w-\phi)} \sin \theta_{e},
$$
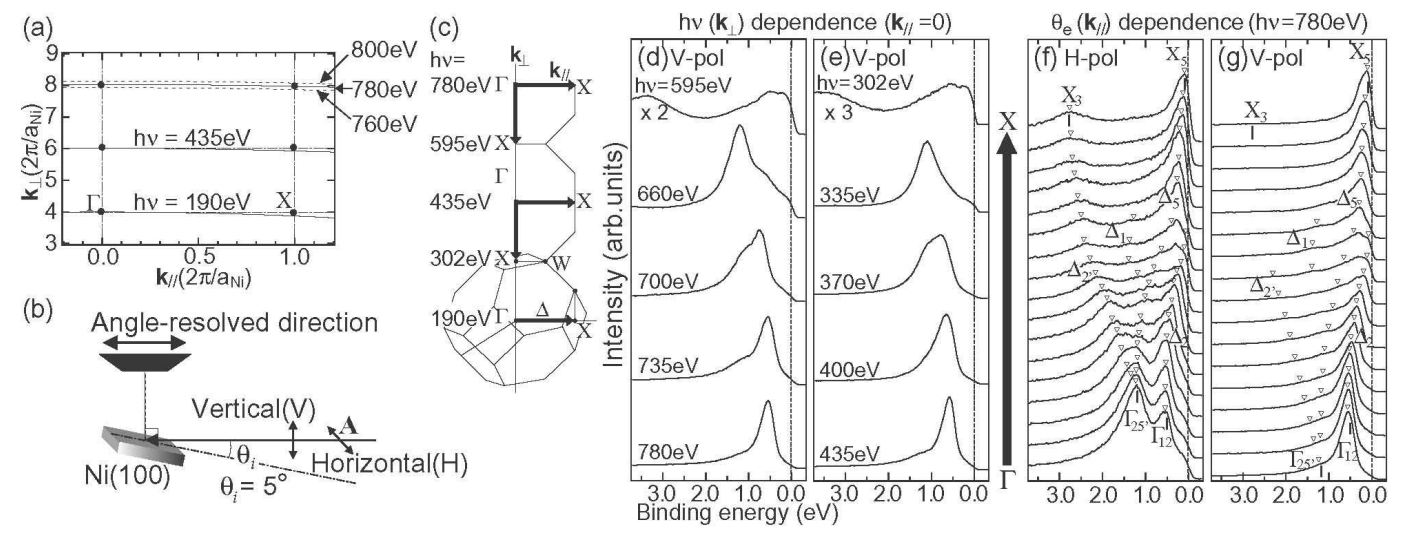

Fig. 1 - (a) $\mathbf{k}_{\perp}$ versus $\mathbf{k}_{\|}$for soft X-ray ARPES of Ni(100) for $h \nu=190,435$ and $780 \mathrm{eV}$ photons by the experimental geometry used(fig. 1(b)). Fig. 1(c) shows the 3-D BZ of Ni(100) alongwith the probed sections. For $\mathbf{k}_{\|}=0$ and $h \nu=(\mathrm{d}) 780-595 \mathrm{eV}$ and (e) 435-302 eV, nearly identical spectra in successive BZs, which are normalized by photon flux, verify the validity of the $\mathbf{k}_{\perp}$ points shown in (a) and (c). Soft X-ray $(h \nu=780 \mathrm{eV})$ ARPES of Ni(100) along $\Gamma-X(\Delta$-line) with (f) $\mathrm{H}$ and (g) $\mathrm{V}$-polarization, where each spectrum is integrated over $0.5^{\circ}$. Peak positions $(\nabla)$ indicate $\operatorname{BDs}\left(\Delta_{2}\right.$, $\Delta_{5}, \Delta_{1}$ and $\left.\Delta_{2^{\prime}}\right)$. 


$$
\hbar \mathbf{k}_{\perp}=\sqrt{2 m\left\{(h \nu-w-\phi) \cos ^{2} \theta_{e}+V_{0}\right\}},
$$

where $\mathbf{k}_{\|}$and $\mathbf{k}_{\perp}$ are parallel and perpendicular components of $\mathbf{k}$, respectively, $\theta_{e}$ is electron emission angle, $\phi$ is work function, and $V_{0}$ is inner potential. The angular resolution of the electron energy analyzer was better than $\pm 0.2^{\circ}$ which corresponds to resolutions of about $\pm 0.014, \pm 0.021$, and $\pm 0.028\left(2 \pi / a_{N i}\right)$ in $\mathbf{k}_{\|}$at $h \nu=190,435$, and $780 \mathrm{eV}$, respectively (eq. (11)). Eqs. (11) and (2) indicate that when ARPES with fixed $h \nu$ is used for measurement of in-plane $\left(\mathbf{k}_{\|}\right)$BDs, $\mathbf{k}_{\perp}$ also changes. The variation of $\mathbf{k}_{\perp}$ can be substantial in ARUPS [28], but diminishes with increasing $h \nu$, making it an advantage. In fig. T(a), we plot $\mathbf{k}_{\perp}$ versus $\mathbf{k}_{\|}$ in units of $\left(2 \pi / a_{N i}\right)$ for $h \nu=190,435$, and $780 \mathrm{eV}$ in the experimental geometry of fig. (1) b), using eqs. (11) and (2) with $V_{0}=9.3 \mathrm{eV}$. Actually, the momentum transfer of the photon is not negligible in soft X-ray ARPES. However, since our experimental geometry is near grazing incidence (fig. 1(b)), the momentum transfer of the photon results in a constant shift of parallel component of the momentum $\mathbf{k}_{\|}$, and $\mathbf{k}_{\perp}$ is negligibly influenced [29]. Therefore, from fig. 1(a) the spectra of $\mathbf{k}_{\|}=0$ at these $h \nu(190,435$, and $780 \mathrm{eV})$ are expected to probe an equivalent $\mathbf{k}_{\perp}$ point $(\Gamma)$ in the 3 -D Brillouin zone (BZ) of Ni(100)(fig. 1(c)). We first validate this by $h \nu$-dependent ARPES of $\mathbf{k}_{\|}=0$ in successive BZs (figs. प(d) and $\mathbf{1}(\mathrm{e})$ ). (The spectra of $\mathbf{k}_{\|}=0$ are identified from the observed band dispersion in the $\left(\theta_{e}\right.$-dependent) ARPES at each photon energy.) The changes in peak positions correspond to $\mathbf{k}_{\perp} \mathrm{BD}$, mainly due to $\Delta_{1}$ band. The similarity of spectra in figs. T(d) and M(e) measured in successive BZs confirm we are at equivalent momentum regions and the $\Gamma$-point can be precisely measured according to eq. (2) even by soft X-ray ARPES. Further, since the difference of $\mathbf{k}_{\perp}$ along $\Gamma-X$ ( $\Delta$-line) of $\mathbf{k}_{\|}$ is already small with $190 \mathrm{eV}$ photons (fig. 1(a)), the in-plane $\mathrm{BD}$ along $\Gamma-X$ can be measured by ARPES with 190, 435, and $780 \mathrm{eV}$ photons. The probing depth or MFP is estimated to be about 5,8 , and $12 \AA$ at $h \nu=190,435$, and $780 \mathrm{eV}$, respectively [9]. Thus we can probe up to the third, fifth, and seventh layer of equivalent regions in the 3-D BZ (figs. 1(a)-(c)).

ARPES with soft X-rays can also lead to indirect transitions caused by the phonon scattering [30]. However, recent ARPES studies with soft X-rays have shown that this can be avoided and the band dispersion can be measured by cooling the sample [31]. Therefore, the present ARPES experiments were performed at $50 \mathrm{~K}$ to minimize the influence of thermal diffuse scattering and hence maximize the direct transition component.

Results and discussion. - In figs. 1(f) and $1(\mathrm{~g})$ we show the ARPES spectra of Ni(100) excited by $780 \mathrm{eV}$ photons with $\mathrm{H}$ - and V-polarization, from $\Gamma$ to $X$. The spectra show angular dependence in $\mathbf{k}$-space from $\Gamma$ to $X$ due to BDs, as marked with triangles. At the $\Gamma$-point in fig. 团 $(\mathrm{f})$, the two peaks correspond to the critical points $\Gamma_{25^{\prime}}$ and $\Gamma_{12}$ at $1.21 \mathrm{eV}$ and $0.51 \mathrm{eV}$ binding energy. Two bands disperse from $\Gamma_{25^{\prime}}$ towards $X$-point and a third band disperses from $\Gamma_{12}$, corresponding to $\Delta_{2^{\prime}}, \Delta_{5}$, and $\Delta_{2}$ bands, respectively (fig. 目f)). Features due to a fourth weak band $\left(\Delta_{1}\right)$ are also barely seen. These BDs are almost consistent with ARUPS results [10-15]. Especially, the energy positions at the high symmetry $\Gamma$ - and $X$ point are very consistent with (spin-integrated) ARUPS results indicated by bars in fig. 11 (f). Fig. 1(g) (V-polarization) reproduces the energy positions of the critical points $\Gamma_{12}$ and $X_{5 \uparrow}$, with consistent BDs as in fig. 1(f) (H-polarization), but enhances the $\Delta_{2}$ band relative to other bands [32]. The results indicate that soft X-ray ARPES can be surely used to obtain BDs of solids. However, there is one important discrepancy in the present data compared with ARUPS results. The $\Delta_{2}$ band crosses the $E_{F}$ in the present spectra and its energy position at the $X$-point, that is $X_{2}$, is evidently above $E_{F}$. This is in contrast with ARUPS studies $[12,14]$ which show that the majority and minority bands of $\Delta_{2}$ symmetry do not cross $E_{F}$, but remain below $E_{F}$ all along the $\Delta$-line. 
As a check of the $\Delta_{2}$ BD measured using $h \nu=780 \mathrm{eV}$, and if truly so, to investigate its deviation from ARUPS results, we measured BDs with $h \nu=435$ and $190 \mathrm{eV}$. In order to clearly see BDs, we plot band maps (BM) (second derivative of raw spectra after smoothing). Figs. 2(a)-(c) show BMs made using the raw spectra shown in Figs. 2(d)-(f) obtained with V-polarization using $h \nu=780,435$, and $190 \mathrm{eV}$, respectively. In fig. 2(a), the $\Delta_{2} \mathrm{BD}$ can be followed unambiguously and the $E_{F}$ crossing is clearly observed (arrow mark), corresponding to the $\Delta_{2 \downarrow}$ minority band. The $\Delta_{2 \uparrow}$ majority BD also can be seen in fig. 2(a) but is more clear in figs. 2(b) and 2(c), with $X_{2 \uparrow}$ located at $0.27 \mathrm{eV}$ binding energy. The spectral intensity very close to the $E_{F}$ crossing of $\Delta_{2 \downarrow}$ (fig. 2(a), just next to the arrow mark), disperses to higher binding energy and is assigned to $\Delta_{5 \downarrow}$. Additional confirmation comes from the $435 \mathrm{eV} \mathrm{BM}$ in fig. 2(b) which shows nearly identical BDs, but for a small change of the $E_{F}$ crossing point in k-space (arrow mark). The changed BD of the $\Delta_{2 \downarrow}$ band with $435 \mathrm{eV}$ photons (fig. [2(b)) results in overlapping the $\Delta_{5 \downarrow}$ band at $E_{F}$, which are separated with $780 \mathrm{eV}$ photons in fig. 22(a). The most important result is obtained with the $h \nu=190 \mathrm{eV}$ BM shown in fig. 22 (c). This BM shows well-resolved majority $\left(\Delta_{2 \uparrow}\right)$ and minority $\left(\Delta_{2 \downarrow}\right)$ bands. Probing just beyond the ARUPS regime changes BDs which are clearly intermediate to the bulk BDs (obtained with $h \nu=435$ and $780 \mathrm{eV}$ ) and surface BDs obtained by ARUPS [10-15]. The $\Delta_{2 \downarrow}$ band crosses $E_{F}$ at $0.57(\Gamma-X)$ in fig. 2(a), at $0.62(\Gamma-X)$ in fig. 2(b), whereas it is $0.76(\Gamma-X)$ in fig. 2(c). The shift of the $E_{F}$ crossing is also seen in the raw ARPES spectra (fig. 22(d)-(f)), which show clear $E_{F}$ crossings with each photon energy(blue curves). The group velocity at $E_{F}$ also changes systematically from $0.62 \mathrm{eV} \AA(190 \mathrm{eV})$, to $0.82 \mathrm{eV} \AA(435 \mathrm{eV})$ and 1.11 eVA $(780 \mathrm{eV})$. Since the $\Delta_{2 \downarrow}$ BD changes systematically, the results indicate that the $\Delta_{2}$ bandwidth and the $\left(X_{2 \uparrow}-X_{2 \downarrow}\right)$ ES is increased in the bulk compared to the ARUPS results.
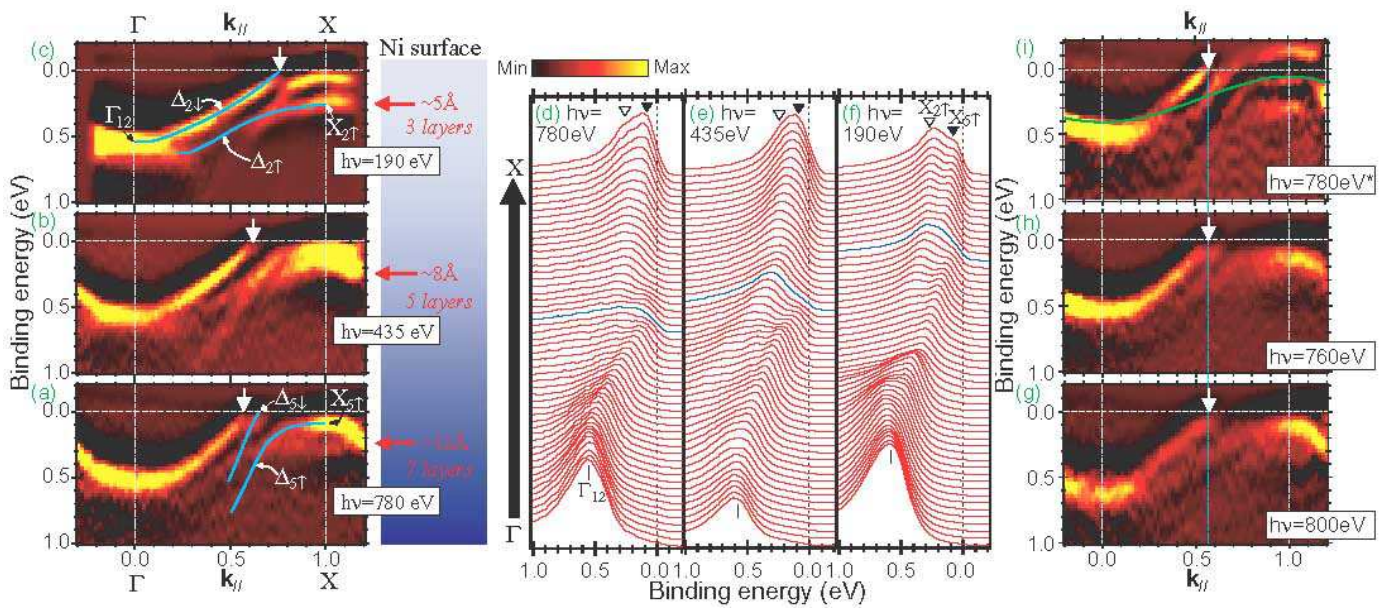

Fig. 2 - Band maps of $\mathrm{Ni}(100)$ along $\Gamma-X$ ( $\Delta$-line) using (a) $h \nu=780 \mathrm{eV}$, (b) $435 \mathrm{eV}$ and (c) 190 $\mathrm{eV}$, with V-polarization. Arrows indicate k-points where the $\Delta_{2 \downarrow}$ band crosses $E_{F}$. The blue lines show the observed band dispersions. The raw ARPES spectra with (d) $780 \mathrm{eV}$ (e) $435 \mathrm{eV}$ ) and (f) $190 \mathrm{eV}$ were used to obtain BMs of (a)-(c). The spectra showing $E_{F}$ crossings are plotted with blue curves in (d)-(f). (g) and (h) show the BMs with $800 \mathrm{eV}$ and $760 \mathrm{eV}$ photons. Fig. 2(i) is obtained as $\mathrm{I}\left(780 \mathrm{eV}^{*}\right)=\mathrm{I}(780)-0.52 \mathrm{I}(435)$ (I: intensity weighted by atomic cross-sections after normalized by photon flux). The green line shows the $\Delta_{2 \downarrow}$ band dispersion summarized from low-energy ARUPS studies and inverse photoemission studies [10-19], which are reproduced by GWA + DMFT [25] calculation. 
From ARUPS the ES is estimated to be $170 \mathrm{meV}$, an anomalously low ES measured only for the $X_{2}$ point on the surface $[12,14,19]$.

We next check how the $E_{F}$ crossing of $\Delta_{2 \downarrow}$ is sensitive to the $\mathbf{k}_{\perp}$-point in Ni band structure. The BMs with $800 \mathrm{eV}$ and $760 \mathrm{eV}$ photons (fig. 2(g) and (h)), which corresponds to $\approx \pm 10 \%$ variation in $\mathbf{k}_{\perp}$ along $\Gamma-X$ (see fig. 团(a)) as compared to data obtained with $780 \mathrm{eV}$ photons (fig. 2(a)) show very similar BDs and also the $E_{F}$ crossing of $\Delta_{2 \downarrow}$ as in fig. 2(a). Therefore, the $\mathrm{BD}$ is insensitive to the $\mathbf{k}_{\perp}$ variations in the range of $\sim 0.1(\Gamma-X)$ for high energies and the change in $E_{F}$ crossing is not due to a change of the $\mathbf{k}_{\perp}$-point.

Since, at any photon energy, the experiments actually measure an integral of the intensity, weighted by an exponential factor for the MFP, we try to extract the bulk BD from ARPES with $780 \mathrm{eV}$ photons. The spectra with $780 \mathrm{eV}$ photons is estimated to roughly contain about $50 \%$ contribution from the top 4-5 layers, which is the probing depth at $h \nu=435 \mathrm{eV}$. The ARPES spectra with $780 \mathrm{eV}$ photons is subtracted by the $435 \mathrm{eV}$ spectra weighted by the exponential factor (0.52) and assuming atomic cross-sections. The result is shown in fig. 22(i) and confirms that the BD seen for bulk Ni are similar to the data of fig. 2 (a). In particular, the separate BDs are more clear at the $X$ point. The data thus prove that the $\Delta_{2 \downarrow}$ BD is different in the bulk compared to surface sensitive ARUPS studies(overlaid as green line).

Thus, the reduced probing depth measurements indicate bulk in-plane BD of $\Delta_{2 \downarrow}$ band systematically connects to the ARUPS results [10-15]. The $\Delta_{2 \downarrow}$ band which is pinned just below $E_{F}$ at $X$-point $\left(X_{2 \downarrow}\right)$ in surface sensitive ARUPS, crosses $E_{F}$ in bulk sensitive ARPES with $X_{2 \downarrow}$ lying above $E_{F}$. This leads to an additional minority spin $X_{2 \downarrow}$ hole pocket. There exists an early spin polarized study by Kisker et al. [33] showing that the $X_{2 \downarrow}$ state lies above $E_{F}$ although later studies have concluded it lies below $E_{F}$. In view of the present results, the larger MFP used but at very low energy ( $4-6 \mathrm{eV}$ ) by Kisker et al. [33] reflects the bulk BS [4]. While de Haas-van Alphen measurements also showed only the $X_{5 \downarrow}$ hole pocket along $\Gamma$ to $X$ [34], magneto-crystalline anisotropy measurements [35] indicated evidence for the additional $X_{2 \downarrow}$ hole pocket.

The layer dependent $\mathrm{BD}$ is coupled to the widening of the $\Delta_{2}$ band width observed in ARPES using high $h \nu$, suggesting weaker electron-electron correlations in the bulk. Theo-

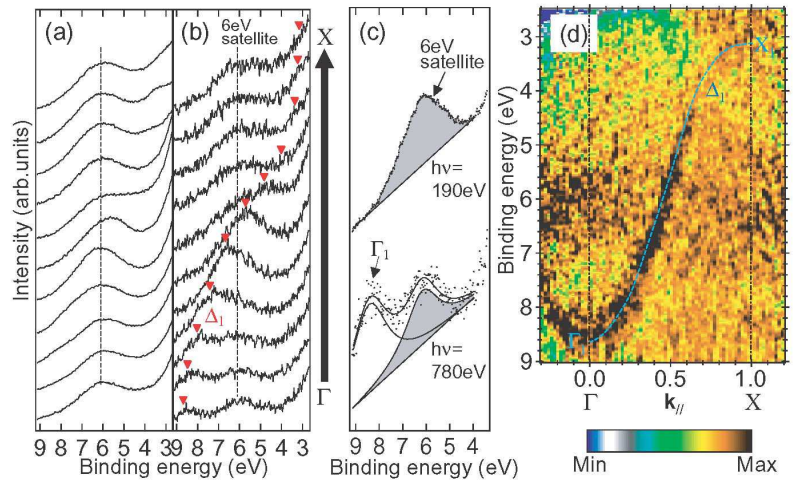

Fig. 3 - ARPES spectra with $h \nu=(\mathrm{a}) 190$ and (b) $780 \mathrm{eV}$, for the $6 \mathrm{eV}$ satellite from $\Gamma$ to $X$, normalized for area under the curve of the entire valence band. Triangles in (b) indicate $\Delta_{1}$ BD obtained from fig. 3(d). (c) A blow-up of $6 \mathrm{eV}$ satellite feature at $\Gamma$-point for the same normalization. Curve fits (solid curves) indicate a reduction of $\sim 20 \pm 4 \%$ in $6 \mathrm{eV}$ satellite intensity for $h \nu=780 \mathrm{eV}$ (shaded area), but no weakening of the correlation energy, consistent with its localized nature. (d) The BM between 2.5 and $9.0 \mathrm{eV}$ binding energy showing $\Delta_{1} \mathrm{BD}$ (broken curve). 
EUROPHYSICS LETTERS

retical calculations which include Coulomb correlations show that $X_{2 \downarrow}$ is located below $E_{F}$ $[14,19,23-25]$ in contrast to s-LDA calculations [22] and the $6 \mathrm{eV}$ satellite is also reproduced [23-25]. Hence, we checked for variations of ARPES spectra in the $6 \mathrm{eV}$ satellite region as a function of $h \nu$ (figs. 3(a) and [3(b)). The ARPES spectra show the $\Delta_{1}$ band dispersing across and overlapping the $6 \mathrm{eV}$ satellite between $\Gamma$ to $X$, as expected from s-LDA calculations [22] and ARUPS studies [10-15]. Even though the $4 s p$ character $\Delta_{1}$ band crossing makes quantification very difficult, an attempt to do so with the $\Gamma$ point spectra (fig. 3(c)), where the $\Delta_{1}$ band is separated, shows a reduction of $\sim 20 \pm 4 \%$ in the $6 \mathrm{eV}$ satellite intensity on increasing $h \nu$ from 190 to $780 \mathrm{eV}$. This reduction in intensity is small and not conclusive but suggestive of reduced correlations in the bulk $[16-18,23]$, consistent with the layer dependent change of $\Delta_{2 \downarrow}$ band dispersion in fig. 2 2 Also, since the electron correlation in Nickel leads to a reduced ES compared to s-LDA calculation [19,25], the reduced correlation is consistent with the enhancement of ES observed at $X_{2}$. Since the $6 \mathrm{eV}$ satellite feature is still observed with $780 \mathrm{eV}$ photons as in angle-integrated X-PES studies [16], it shows that ARPES is necessary to check for the bulk to surface changes in the valence BS. In fig. 3 (d), we plot a BM for 2.5 to $9.0 \mathrm{eV}$ binding energy and $h \nu=780 \mathrm{eV}$, where $\Delta_{1} \mathrm{BD}$ is clearly seen, consistent with ARUPS results. We have also confirmed that the $\Delta_{1} \mathrm{BD}$ is similar to that obtained by applying the sum rule as discussed in ref. [36].

Another important point to note is that the $\Delta_{5}$ and $\Delta_{2^{\prime}}$ (fig. If(f)) BDs do not change with $h \nu$ i.e. as a function of probing depth. Since $\Delta_{5}$ and $\Delta_{2^{\prime}}$ band originate in $t_{2 g}$-type states, it is not modified by electron correlations like the $e_{g}$-derived states [23]. Even between the $e_{g}$-derived states, the $\Delta_{2}$ band $\left(d_{x^{2}-y^{2}}\right)$ possibly shows more layer dependent correlation than the $\Delta_{1}$ band $\left(d_{z^{2}-r^{2}}\right)$ since the $\left(d_{x^{2}-y^{2}}\right)$ orbital extends outwards along the surface normal direction (100). This suggests similarity to recent results showing varying correlations between the $t_{2 g}$-orbitals of $\left(d_{x y}\right),\left(d_{x z}\right)$, and $\left(d_{y z}\right)$ symmetry for correlated oxides [5].

Conclusion. - In conclusion, tunable soft X-ray polarization dependent ARPES makes it possible to resolve the changes in BDs with specified symmetry and layers. We observe layer dependent $\Delta_{2 \downarrow}$ band dispersion, which leads to an additional minority spin $X_{2 \downarrow}$ hole pocket. However, the $\Delta_{5}$ and $\Delta_{2^{\prime}}\left(t_{2 g}\right)$-symmetry bands are not affected by the existence of the surface. Thus, the observed bulk versus surface difference in behavior of the electronic states depends on the band symmetry. The results show layer dependent BD changes, suggesting correlation effects and ES being coupled to changes in the probing depth, and the importance of Coulomb correlations in theoretical calculations for the surface electronic BS. Soft X-ray ARPES is thus shown to be a very important tool to study layer dependent BD of 3-D solids.

\section{$* * *$}

We thank Drs. H. Ohashi, Y. Tamenori, T. Ito, and P. A. Rayjada for help with experiments and Profs. A. Fujimori, T. Yokoya, A. Kotani, M. Taguchi, M. Usuda, T. Jo, and Dr. K. Horiba for valuable discussions.

\section{REFERENCES}

[1] Segovia P., Purdie D., Hengsberger M. And Baer Y., Nature, 402 (1999) 504.

[2] Valla T. et al., Nature, 417 (2002) 627.

[3] Lanzara A. et al., Nature, 412 (2001) 510.; KAminski A. et al., Nature, 416 (2002) 610.; Yusof Z. M. et al., Phys. Rev. Lett., 88 (2002) 167006.

[4] Plummer E. W. and Eberhardt W., Adv. Chem. Phys., 49 (1982) 533. 
[5] Liebsch A., Phys. Rev. Lett., 90 (2003) 096401.; Schwieger S., Potthoff M. And Nolting W., Phys. Rev. B, 67 (2003) 165408.

[6] Rader O. et al., Europhys. Lett., 39 (1997) 429.

[7] Sekiyama A. et al., Nature, 403 (2000) 396.

[8] Maiti K., Mahadevan P. And Sarma D. D., Phys. Rev. Lett., 80 (1998) 2885.; Mo S.-K. et al., Phys. Rev. Lett., 90 (2003) 186403.

[9] Tanuma S., Powell C. J. and Penn D. R., Surf. Interface anal., 11 (1988) 57.

[10] Himpsel F. J., Knapp J. A. and Eastman D. E., Phys. Rev. B, 19 (1979) 2919.

[11] Eberhardt W. and Plummer E. W., Phys. Rev. B, 21 (1980) 3245.

[12] Heimann P., Himpsel F. J. and Eastman D. E., Solid State Commun., 39 (1981) 219.

[13] Mårtensson H. and Nilsson P. O., Phys. Rev. B, 30 (1984) 3047.

[14] Bünemann J., Gebhard F., Ohm T., Umstätter R., Weiser S., Weber W., Claessen R., Ehm D., Harasawa A., Kakizaki A., Kimura A., Nicolay G., Shin S. and Strocov V. N., Europhys. Lett., 61 (2003) 667.

[15] Kakizaki A. et al., Phys. Rev. Lett., 72 (1994) 2781.; Greber T., Kreutz T. J. And OsterWAlder J., Phys. Rev. Lett., 79 (1997) 4465.

[16] Hüfner S. et al., Solid State Commun., 11 (1972) 323.

[17] Guillot C. et al., Phys. Rev. Lett., 39 (1977) 1632.

[18] Kinoshita T. et al., Phys. Rev. B, 47 (1993) 6787.; Sinkovic B. et al., Phys. Rev. Lett., 79 (1997) 3510.

[19] Donath M., Surf. Sci. Rep., 20 (1994) 251.

[20] Slater J. C., Phys. Rev., 49 (1936) 537.

[21] Stoner E. C., Proc. Roy. Soc. A, 165 (1938) 372.

[22] Wang C. S. and Callaway J., Phys. Rev. B, 15 (1977) 298.

[23] Liebsch A., Phys. Rev. Lett., 43 (1979) 1431.

[24] Lichtenstein A. I., Katsnelson M. I. and Kotliar G., Phys. Rev. Lett., 87 (2001) 067205.

[25] Biermann S., Aryasetiawan F. and Georges A., Phys. Rev. Lett., 90 (2003) 086402.

[26] Ohashi H. et al., Nucl. Instrum. Meth. A, 467-468 (2001) 529.

[27] Tanaka T. and Kitamura H., J. Synchrotron Radiation, 3 (1996) 47.

[28] It is also possible to measure $\mathbf{k}_{\|}$band dispersions in the constant final state (CFS) mode without $\mathbf{k}_{\perp}$ variation compared to the energy distribution curve (EDC) mode described, even with ARUPS, see e.g. Photoemission in Solids, edited by Cardona M. and Ley L., Vol. I (SpringerVerlag, Berlin) 1978, p. 261.

[29] This is understood from the measured spectra which show constant shifts in $\mathbf{k}_{\|}$by $0.22(\Gamma-X)$, while the $\mathbf{k}_{\perp}$ negligibly shifts, for $780 \mathrm{eV}$. Similarly, for $h \nu=435 \mathrm{eV}$ and $190 \mathrm{eV}$, constant $\mathbf{k}_{\|}$ shifts of $0.12(\Gamma-X)$ and $0.05(\Gamma-X)$ were determined. The shift of $\mathbf{k}$ is identified from accurate determination of the high symmetry points $\Gamma$ and $X$.

[30] Shevchik N. J., Phys. Rev. B, 16 (1977) 3428; 20 (1979) 3020; Hussain Z., Kono S., Peterson L. G., Fadley C. S. and Wagner L. F., Phys. Rev. B, 23 (1981) 724.

[31] Hofmann Ph. et al., Phys. Rev. B, 66 (2002) 245422.; Nielsen M. B., Li Z., Lizzit S., Goldoni A. And Hofmann Ph., J. Phys. Condens. Matter, 15 (2003) 6919.

[32] In fig. 1] (d) and (e), $\Delta_{1}$ band should be mainly observed due to dipole selection rules and the intensity decrease at $X$-point seen in the figure is caused by the $\Delta_{1}$ band moving above $E_{F}$, as also expected from ARUPS. In fig. 11(f) and (g), on the other hand, bands with all symmetries along $\Delta$ can be observed because of the measurement of the in-plane band dispersion, and the relative intensity of each band is governed by matrix elements. The differences in spectra is also attributed to the different volumes sampled with the different photon energies used.

[33] Kisker E., Gudat W., Kuhlmann E., Clauberg R. and Campagna M., Phys. Rev. Lett., 45 (1980) 2053.

[34] Tsui D. C. and Stark R. W., Phys. Rev. Lett., 17 (1966) 871.

[35] Gersdorf R., Phys. Rev. Lett., 40 (1978) 344.

[36] Freund H. -J., Eberhardt W., Heskett D. and Plummer E. W., Phys. Rev. Lett., 50 (1983) 768. 